\title{
Reflections on the therapy assisted by animals in palliative care
}

\begin{abstract}
The relationship between humans and animals has been a subject of discussion over the years. The Animal-Assisted Therapy encounter space increasingly present therapeutic support and studies reveal reduction of the level of anxiety, stress and pain relief, besides corroborating for the harmonization of the professional environment It has been discussed the benefits of animal assisted therapy in palliative care and its potential for promotion and effective improvements in patient care. Thus, this study aims to reflect on this current theme and the perspectives and contributions of Animal Assisted Therapy during the care process in palliative care.
\end{abstract}

Keywords: palliative care, animal assisted therapy, humanization of assistance
Volume I Issue 2 - 2017

Ronny Anderson de Oliveira Cruz

Department of Nursing, University Center of João Pessoa, Brazil

Correspondence: Ronny Anderson de Oliveira Cruz, University Center of João Pessoa - UNIPÊ, Br 230 - Km22 S/N, UNIPÊ Campus - 58053-000 - JOAO PESSOA-PB-Brazil, Email ronnyufpb@gmail.com

Received: March 20, 2017| Published: June 21, 2017
Abbreviations: AAT, animal assisted therapy; PC, palliative care

\section{Introduction}

Palliative care (PC) is a care approach that aims to improve the quality of life of patients and their families, with clinical condition that threatens Life and quality of life, through prevention, evaluation and treatment of pain and of psychosocial and spiritual support. Conceptual changes in the field of health are observed, especially those that started from the extension of its concept through the establishment of new guidelines proposed by the World Health Organization, where health ceases to be an absence of disease to become a state of wellbeing physical, psychic, social and spiritual. ${ }^{1,2}$

The advancement of technologies has provided the development of new therapeutic modalities and thus improvements in the care of those in need of palliative care. In the meantime, the care traditionally performed in environments such as those in intensive care facilities has been adapted to its institutional protocols, corroborating the use of non-pharmacological interventions, increased physiotherapy, speech therapy, psychology, relaxation techniques and music therapy. ${ }^{3}$

The increase in life expectancy has led to an increase in the number of individuals with chronic diseases, corroborating an expressive demand for attention where the cure, in some cases, is no longer possible. ${ }^{2}$ Animal-Assisted Therapy (AAT) meets this concept and has been used for several years as a complementary measure to drug treatment, aiming at the improvement and well-being of the patient. Its efficacy has been proven through studies worldwide, in different areas such as pediatrics, geriatrics, psychiatry and more recently, in palliative care, bringing both physical and mental health benefits of sick people. ${ }^{4}$ Thus, this study aims to reflect on the potentialities and contributions of TAA during the implementation of the palliative care process.

\section{Methods}

A descriptive study, type reflexive analysis, supported by studies from the Medical Literature Analysis and Retrieval System Online database (MEDLINE), Latin American and Caribbean Literature in Health Sciences (LILACS), Scientific Electronic Library online
(SCIELO), Through the descriptors "palliative care" and "Assisted therapy by animals" from February to May 2017. The inclusion criteria were complete studies, in the Portuguese and English languages, published in the last 5 years and that included information about the Guiding question: what are the contributions of animal assisted therapy to palliative care?. Those of exclusion were dissertations and theses. For analysis, the technique of discursive textual analysis was used in which the sense nuclei composing a communication were discovered, whose presence or frequency adds significant perspectives to the object of study in question. The notion of the subject is associated with an affirmation that relates to a certain subject, and can be presented by a word, phrase or idea. ${ }^{5}$

\section{Discussion}

Palliative care consist of patient-oriented practices, where it is understood that the disease is, progressive, irreversible and not responsive to established treatment. Thus, professionals must provide quality and comprehensive care, aiming for comfort in the final moments of life. The paradigm shift between healing and palliative care is a process that depends on professionals working in the field and academic institutions, as well as professional knowledge for this interface of care. Therefore, treatment based on professional training and qualification governed by scientific principles is essential. ${ }^{6}$

It should be noted that at various times the health professional feels distressed and has a sense of failure for not achieving the goal proposed from the conservative abrodation of curative medicine, and the patient feels powerless because he does not have the necessary support in such a situation. fragile. For those professionals who do not present any degree of training in palliative care tend, over time, to create an affective withdrawal from the patient. ${ }^{7}$

The relationship between human beings and animals has been discussed for a long time. Animals arouse the interest, attention and affection of people, from childhood to old age, playing an important role in their lives, either as pets and company or as a co-therapist. ${ }^{8}$

In the health area there have been reports of the importance and benefits arising from this relationship. There are already recognized practices that use animals as auxiliary tools in promoting health improvement, rehabilitation, and palliation. The visit of animals in 
the hospital establishes a relaxed atmosphere among professionals, children and family, being recognized with an effective strategy of humanization of care. ${ }^{9}$

Contact with animals helps release the hormones that bring the feeling of well being, increasing the production of endorphin and serotonin reducing cortisol rates. In patients undergoing cancer treatment, which involves chemotherapy and radiation therapy, the side effects can be overwhelming, and the animals help to deflect the disease. In addition, it increases motor capacity, improves the immune system, decreases the symptoms of depression, reduces anxiety and lowers blood pressure, causing medication intake to decrease. ${ }^{10}$

The evidence of TAA in palliation reflects in the social, emotional and psychological spheres for adults. In a study among cancer patients, it was found that while participants reported that TAA sessions helped them to alleviate anxiety and served as a good distraction in the hospital setting, statistical significance was not found for these results compared to reading sessions And human visits.

\section{Conclusion}

From this reflection it was possible to perceive that although the palliative care environment has evolved in relation to aspects of humanization and the use of new therapeutic approaches, the presence of animals is still full of doubts and resistance. It is necessary to increase the studies and the level of evidence in order to support and present the benefits of this type of care. Studies even with a timid number of publications on the subject already point to the well-being of the patient in palliation that may have an animal being part of their care process. Apart from this aspect it is still able to reduce the stress and anxiety of the health team. It is a fact that there is still a long way to go for acceptance of activities involving animals in the hospital and palliation, but the viability is clear.

\section{Authors' Contributions}

The author was responsible for the construction and critical reading of the manuscript.

\section{Acknowledgements}

None.

\section{Conflict of interest}

The author declares no conflict of interest.

\section{References}

1. Duarte IV, Fernandes KF, Freitas SC. Home palliative care: considerations about the role of the family caregiver. Rev SBPH. 2013;16(2):73-88.

2. WHO. Definition of Palliative Care. World Health Organization, Geneva, Switzerland: Springer; 2014.

3. Fonseca AC, Mendes Junior WV, et al. Palliative care of elderly patients in intensive care units: a systematic review. Rev Bras Ter Intensiva. 2012;24(2):197-206.

4. Crippa A, Feijó AGS. Actividad assistida por animales. Rev latinoam bioet. 2014;14(1):14-25.

5. Moraes R, Galiazzi MC. Análise textual discursiva. Rev Ijuí: Editora Unijuí. 2011;2(1):117-128.

6. Silveira NR, Nascimento ERP, Rosa LM, et al. Palliative care and the intensive care nurses: feelings that endure. Rev Bras Enferm. 2016;69(6):1012-1019.

7. Costa AP, Poles K, Silva AE. Palliative care education: experience of medical and nursing students. Interface (Botucatu). 2016;20(59):10411052.

8. Reed R, Ferrer L, Villegas N. Natural healers: a review of therapy and activities assisted by Animals as complementary treatment for chronic diseases. Rev Latino-Am Enfermagem. 2012;20(3):1-7.

9. Ministério da Saúde (BR). Humaniza SUS: Política Nacional de Humanização. Distrito Federal, Brasília: Springer; 2015. p. 1-157.

10. INCA. Rede Câncer. Instituto Nacional de Câncer José Alencar Gomes da Silva, Rio de Janeiro: Springer; 2016;34:10-15. 